\title{
Control of Optical Properties by the Stepwise Chemical and Plasma Spray Treatment of Polycarbonate
}

\author{
Bup Ju Jeon* \\ Department of Energy \& Environmental Engineering, Shinhan University, Gyeonggi-do 483-777, Republic of Korea
}

Received November 21, 2018; revised December 2, 2018; accepted December 3, 2018

\begin{abstract}
Using the Polycarbonate (PC) polymer sheet, the change in optical properties was compared by changing the surface shape with the primary chemical surface treatment and plasma spray treatment. Plasma treatment affects surface composition changes and has a small effect on surface structure changes. Changes in surface structure are important to control the optical characteristics of the PC polymer sheet. Thus, it was possible to control changes in surface composition and changes in surface structure through secondary plasma treatment after primary chemical treatment. The primary chemical treatment had a small effect on changes in the chemical composition of the surface, but the surface roughness was influenced by swelling. The secondary plasma spray treatment affected optical properties changes, which allowed control of changes in surface structure after primary chemical treatment. At the same time after the first chemical treatment, the secondary plasma treatment was easy to control of optical properties by changing the surface structure as well as the surface properties due to changes in chemical composition by surface cross-linking reaction.
\end{abstract}

Keywords: Polycarbonate polymer sheet, Chemical treatment, Plasma treatment, Surface roughness, Optical properties

\section{Introduction}

Plastic surface treatment provides functionalities such as anti-fouling and self-cleaning. It also can be used as a cover material of display, lens, and optical filters by changing the light transmittance and the hazes [1-4]. To give functionality to plastic surfaces, chemically introducing functional groups on surfaces or physically altering the surface structure is being used. Surface treatment of polymer has been used primarily to change the surface properties and improve adhesion with other substances. Changes in surface structure can form nano particles on a surface or be functional-coated. Surface treatment method is used through plasma of DC or Radio Frequency (RF) [59]. Polycarbonate (PC) is applied to various fields such as optical and display materials due to its high performance light transmittance and mechanical strength. In particular, there are many applications since the variation of optical properties due to surface treatment is free. Various studies were conducted to change the characteristics of the PC surface, among which studies were conducted to improve the surface properties by incoporation of the nano particles after swelling the polymer surface with a solvent for

*Corresponding author

E-mail: bjjeon@shinhan.ac.kr chemical treatment [9-11].

In this study, we compared the variation in optical properties by using PC polymer sheets to change the optical properties through primary chemical surface treatment and then using plasma spray to change the surface shape of the microstructure to the optical properties.

\section{Experimental details}

\section{Surface treatment of PC sheet}

For the adjustment of incident light from transmittance and reflection on the surface of the PC polymer plate, after chemical treatment first using acetone, the second treatment was performed with plasma spray. The PC used in the experiment is a sheet with a transmittance of $92 \%$ that is $1 \mathrm{~mm}$ thick and $150 \mathrm{~mm} \times 150 \mathrm{~mm}$ in dimension. Chemical treatment was first performed for primary surface treatment. To change the structure of the surface, acetone (\% of company name) was used and the concentration was changed to react for a period of time with the volume fraction between 0.750 and 0.875 . To maintain a uniform concentration, $500 \mathrm{rpm}$ was stirred to a constant temperature of $25^{\circ} \mathrm{C}$. After the first surface treatment, it was washed with distilled water to stop the reaction and dried by air blowing. After air blowing, the 


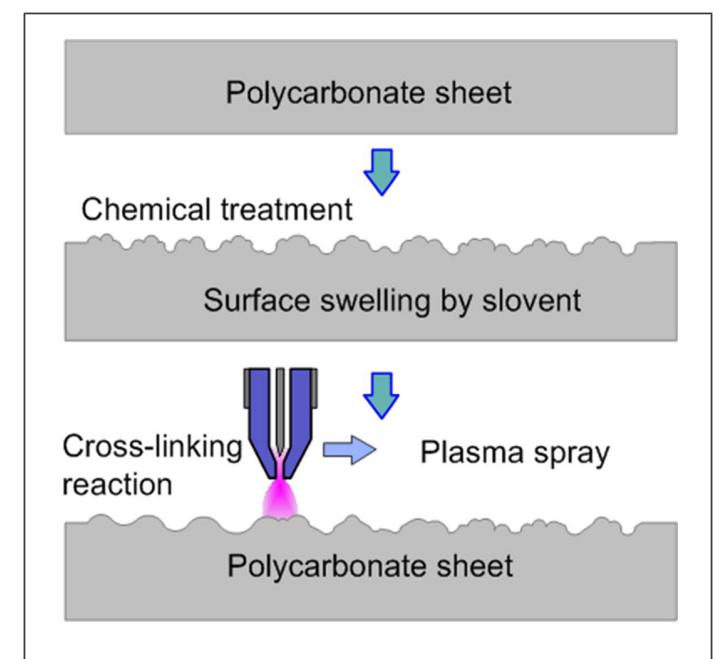

Figure 1. Schematic diagram for surface treatment of the polycarbonate sheet by chemical method and plasma spray method.

secondary plasma surface treatment was performed after a 24-hour at atmospheric pressure condition to remove some remaining solvents by surface swelling. For plasma surface treatment, a atmospheric-pressure plasma with a voltage of up to $4 \mathrm{kV}$ and a frequency of $40 \mathrm{kHz}$ configuring a DC$\mathrm{AC}$ inverter was used. The air pressure for surface treatment is $180 \mathrm{MPa}$ and the substrate and distance are kept constant at $2 \mathrm{~cm}$ for surface treatment by uniform plasma density. Line speed of plasma spray was treated at $2 \mathrm{~cm} / \mathrm{sec}$. Figure 1 shows a schematic diagram that chemically processes the surface structure using a PC sheet and changes the surface structure with a cross-link reaction by using plasma spray.

\section{Analysis}

Physical and optical surface characteristics of substrates were investigated after the primary chemical treatment of polymer sheet. First, surface contact angle measuring instruments were used under $20^{\circ} \mathrm{C}$ conditions using water to check chemical treatment and hydrophobic properties by plasma spray. The contact angle was measured three times and the average value was used. To check the optical properties, the visible light transmittance was measured using Jasco's UV-visible spectrum-photometer (model V730). Using the rhopoint Gloss meter (model iQ 3 angle), the reflection haze was measured. The surface roughness of the primary chemical treatment and secondary plasma spray treated substrates affected the optical transmittance and the reflection haze, so a change in surface roughness at each stage was measured with a portable light measuring device (Model SJ10). X-ray phosphor spectroscopy (XPS, model PHI 58000) analysis was performed to confirm changes in surface composition of PC sheet before and after he surface treatment.
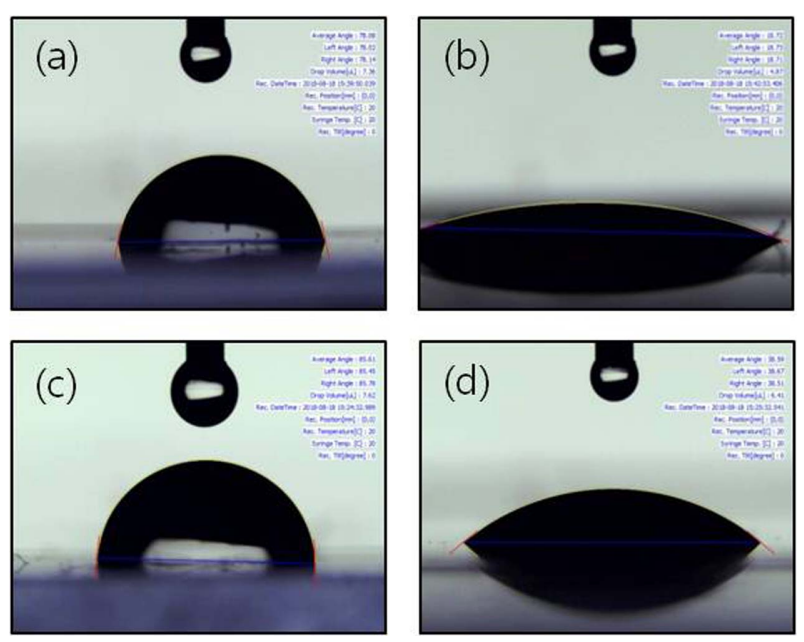

Figure 2. Contact angle images for droplets water on polycarbonate sheet before and after chemical and plasma spray treatment. (a) Pristine PC, (b) plasma spray treated PC, (c) chemical treated $P C$ at acetone volume fraction 0.83 , and (d) plasma spray treated Polycarbonate after chemical treatment at acetone volume fraction 0.83 .

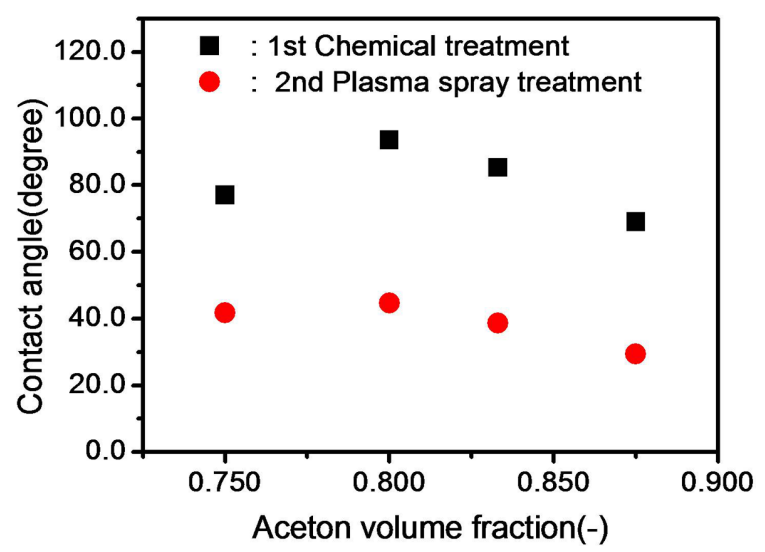

Figure 3. Comparison of contact angle of chemical treatment $P C$ and plasma spray treatment $P C$ after chemical treatment with the solvent volume fraction.

\section{Results and discussion}

Figure 2 shows an image of droplets water on the PC sheet measuring the contact angle using water to identify changes in surface properties for $6 \mathrm{~min}$ of surface reaction and before and after plasma spray under conditions forming a volume fraction of acetone 0.8. Figure 2(a) shows the contact angle of the pristine PC sheet with a contact angle of $78.08 \pm 0.6^{\circ}$. Figure 2(b) measured the contact angle after surface treatment with plasma spray directly on the pristine PC board when the value was shown to be $18.72 \pm 1^{\circ}$. Figure 2(c) shows that the contact angle of a chemical treated specimen for six minutes under the conditions of 0.83 , which is a volume fraction of acetone, is $85.61 \pm 1.5^{\circ}$ lower than that of a plasma specimen. Figure 2(d) shows the contact angle of the 

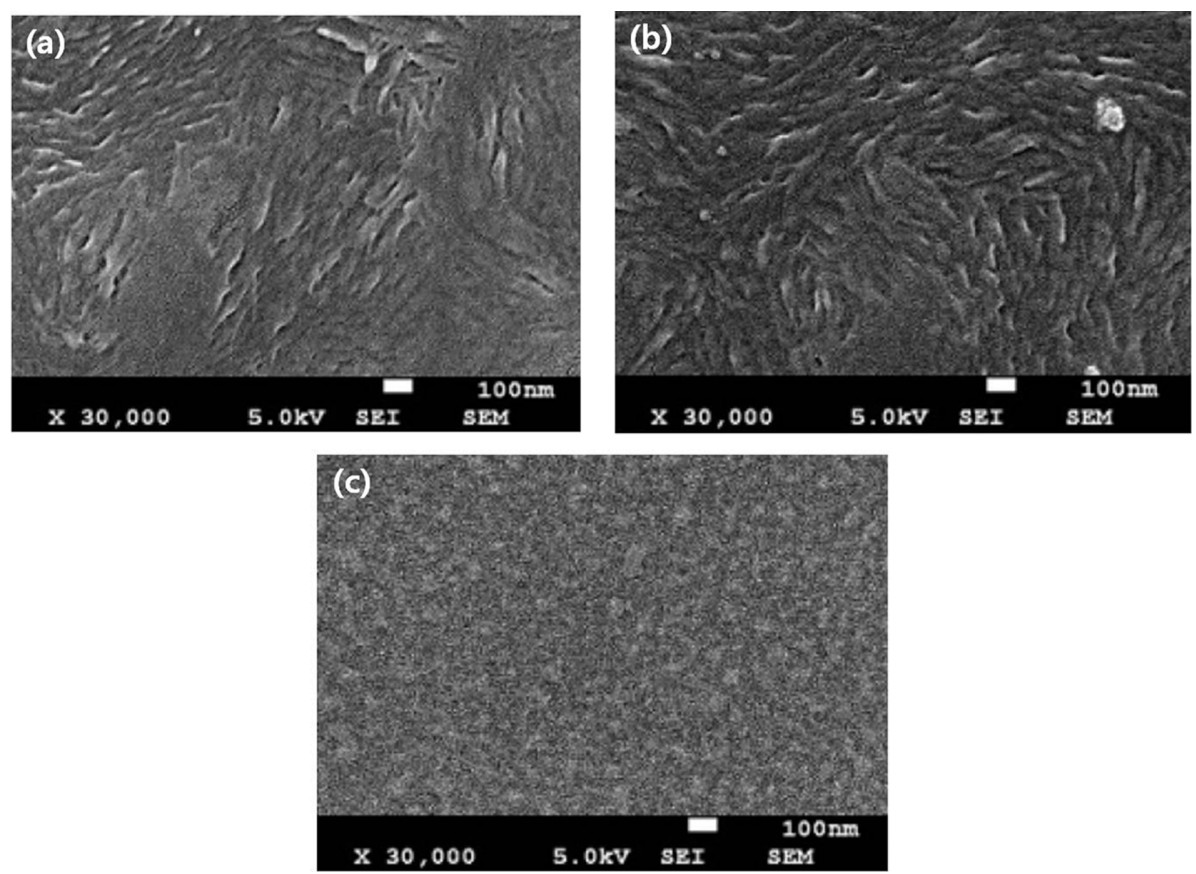

Figure 4. SEM images of the polycarbonate sheet obtained by (a) pristine PC, (b) chemical treatment PC, and (c) plasma spray treatment after chemical treatment.

secondary surface treatment by a plasma spray after the primary chemical treated specimen shown in Fig. 2(c), at $38.59 \pm 0.8^{\circ}$. Figure $2(\mathrm{~d})$ shows the contact angle of the secondary surface treatment by a plasma spray after the primary chemical treated specimen shown in Fig. 2(c). The contact angle was then $38.59 \pm 0.8^{\circ}$. As shown in the image of Fig. 2, direct plasma surface treatment produces $\mathrm{C}=\mathrm{O}$ and $\mathrm{C}-\mathrm{O}-\mathrm{C}$ groups as a substitute for surface crosslinking reactions and oxygen, more changes can be identified than chemical treatment.

Figure 3 shows the change in the contact angle of a specimen by surface treatment of secondary plasma spray after the first chemical treatment according to changes in the volume fraction of acetone. In the case of a volume fraction of 0.75 treated chemically, the contact angle of the specimen was $77.12^{\circ}$, not much different from the pristine PC sheet. The contact angle tends to increase as the amount of acetone increases, and it is certain that appears to removed of the oxygen binding layer present on the surface by etching. Under a volume fraction of acetone at 0.875 it appears to be reduced again, influenced by changes in surface roughness rather than by the substituent group of substrate surfaces. In the case of samples treated with the second plasma spray after the first chemical treatment, the contact angle decreased steadily due to increased hydrophilic by oxygen group replaced on the surface. Contact angles were also influenced by surface roughness by chemical treatment, indicating a tendency similar to chemical treatment according to the volume fraction of acetone.

Figure 4 shows the images of FESEM measurements of surface shapes of primary chemical surface treatments and
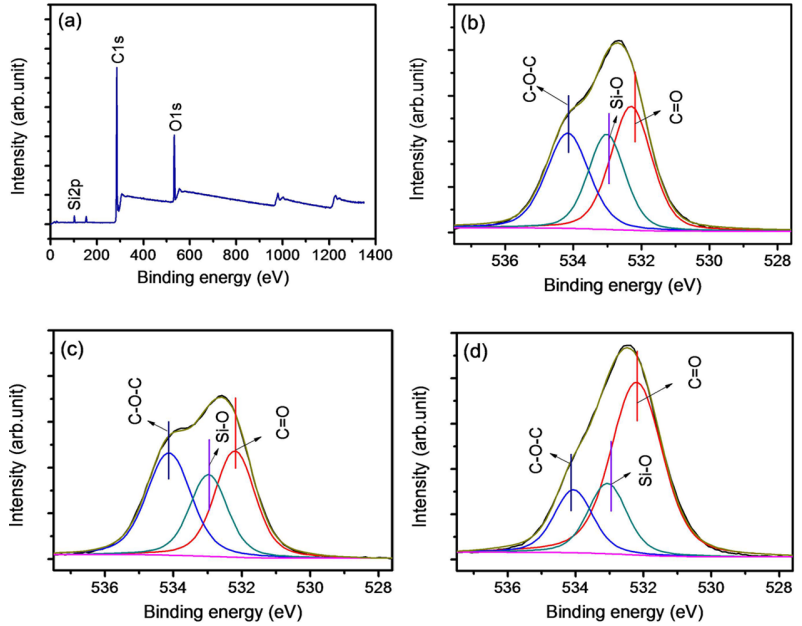

Figure 5. XPS analysis of PC. (a) Wide scan, peak separation of (b) pristine PC, (c) chemical treatment, and (d) plasma spray treatment of O1s peaks.

secondary plasma spray surface treatments. From the primary chemical treatment sample and the secondary plasma spray surface processing image comparison, the grain tissue of a chemical treatment sample was shown big. In case a sample of chemical treatment is again plasma treated, the grain tissue shows a small and dense image. This is caused by the swelling and dissolved of the PC surface by acetone, resulting in a rough and large surface tissue. On the other hand, in case plasma spray treatment, it appears to have formed a dense tissue due to surface cross-link reactions.

Figure 5 shows the XPS analysis results to confirm changes in surface chemical structures on the pristine PC, 


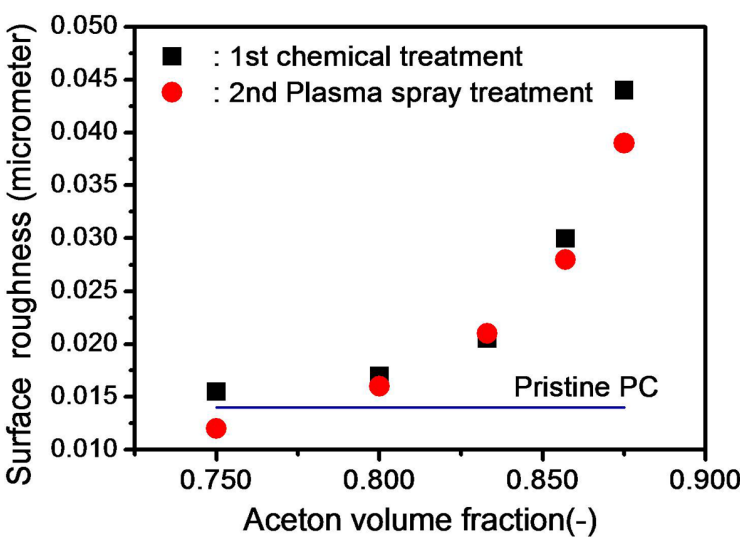

Figure 6. Comparison of the surface roughness of chemical treatment $\mathrm{PC}$ and plasma spray treatment $\mathrm{PC}$ after chemical treatment with the solvent volume fraction.

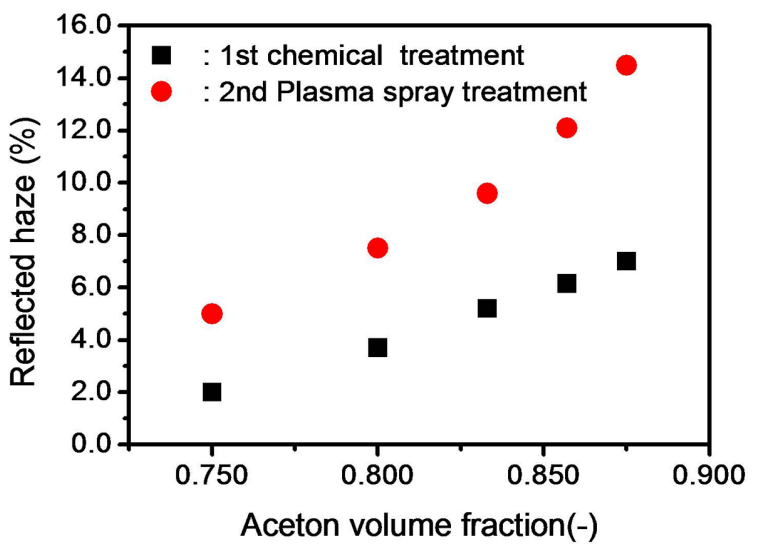

Figure 7. Comparison of the reflecttion haze of the polycarbonate sheet by chemical treatment and plasma spray treatment PC after chemical treatment with the solvent volume fraction.

primary chemical treatment, and secondary plasma spray treatment samples. In general, Fig. 5(a) shows the result of a wide scan of the PC sheet from 0 to $1300 \mathrm{eV}$. As shown in the Fig. 5(a), PC sheet can identify the main peaks of $\mathrm{C} 1 \mathrm{~s}, \mathrm{O} 1 \mathrm{~s}$ and $\mathrm{Si} 2 \mathrm{p}$ which consists of $\mathrm{SiO}_{2}$ and organic carbon. Generally, when polymers such as PET or PE are exposed to plasma condition, the surface of the substrate is generated by carbonyl or ester groups, which changes the surface characteristics [12]. Also, plasma treatment can affect changes in surface composition, but it is not easy to change the haze by plasma treatment because of its small impact on surface structure changes. Figs. 5(b) (d) indicates peak separation of O1s peaks at 528 to $537 \mathrm{eV}$ for the pristine PC, primary chemical treatment, and secondary plasma samples. In Fig. 5(b), it can be seen that the surface composition of the pristine $\mathrm{PC}$ consists of $\mathrm{C}=\mathrm{O}$ combination at $532.20 \mathrm{eV}, \mathrm{SiO}$ combination at $533.06 \mathrm{eV}$, and metal carbonate or C-O combination at $534.05 \mathrm{eV}$. In Fig. 5(c), which represented the primary chemical treatment sample, surface showed a slightly increase in C-O-C bonds at $533.06 \mathrm{eV}$ by surface chemical reaction and was generally similar to the pristine PC. Figure 5(d) shows separated peaks
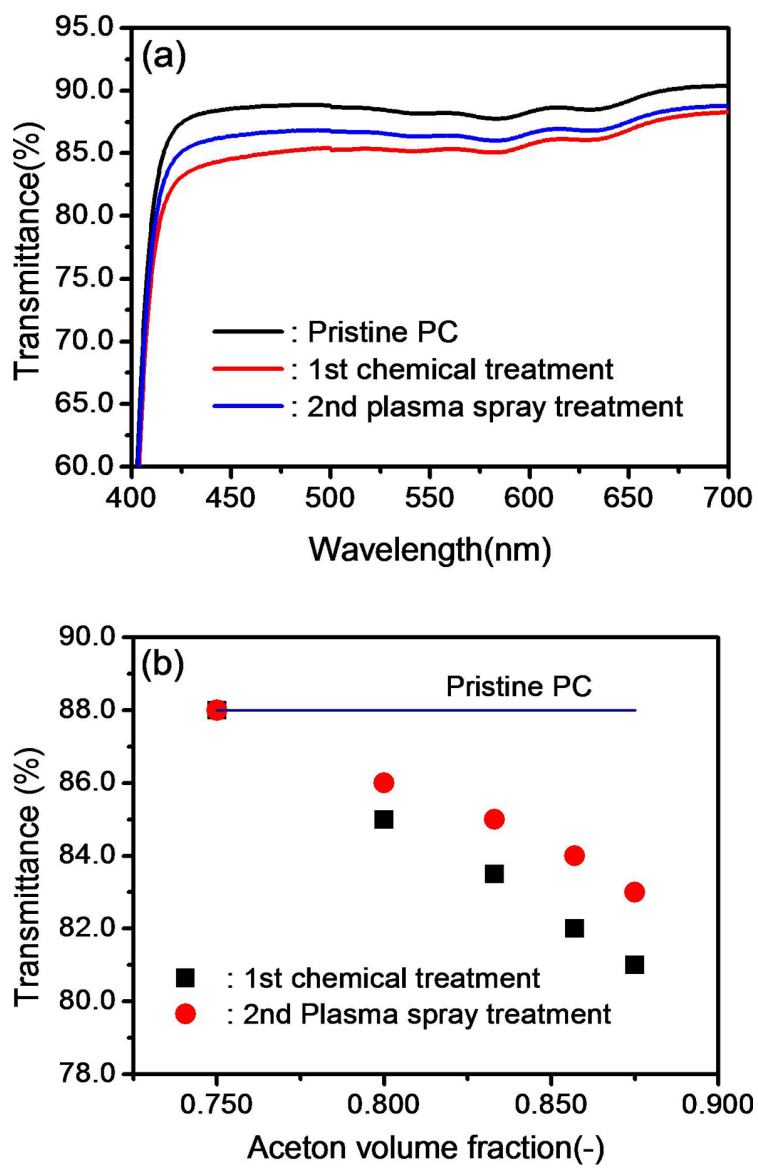

Figure 8. (a) Optical transmission at visible region and (b) transmittance at $550 \mathrm{~nm}$ of chemical treatment and plasma spray treatment with the solvent volume fraction.

at O1s the plasma spray treated sample. The plasma spray test specimen shows that $\mathrm{C}=\mathrm{O}$ combination increased compared to the C-O-C combination by oxygen radical with high energy of plasma state. Therefore, in case of plasma spray treatment, it is possible to verify that the properties have changed with different surface composition like an amorphous graphene carbon than the chemical treatment and the pristine $\mathrm{PC}$.

Figure 6 shows the comparison of the surface roughness by plasma spray treatment and the chemical surface treatment. For a relative comparison of surface roughness, the illumination of the pristine $\mathrm{PC}$ was found to be $0.014 \mu \mathrm{m}$. For a relative comparison of surface roughness, the roughness of the pristine PC was measured and the result was $0.014 \mu \mathrm{m}$. First of all, changes in the surface roughness of the primary chemical treated specimen depend on the content of acetone and tend to increase rapidly as the volume fraction of acetone increases. For samples treated with secondary plasma, the surface roughness was lower than the pristine $\mathrm{PC}$ under 0.75 , with a lower volume fraction of acetone. As the content of acetone increases, the surface roughness difference between the primary chemical and secondary plasma spray treated specimen increases slightly. This appears to be due 
to the increased surface shape due to the cross-link reactions caused by plasma treatment as the degree of swelling of chemically treated surfaces increases.

Figure 7 shows the reflection haze of samples according to changes in acetone concentration in the primary chemical treatment and plasma spray treatment. The reflection haze indicates an inverse relationship with transmission haze [13]. Reflective haze tends to increase with acetone concentrations in primary chemical treatment. In the case of secondary plasma spray treatment, reflective haze was higher than the primary chemical treatment, but tends to be similar. Overall, it is believed that reflective haze has increased proportionally with the effects of surface roughness by cross-linking reaction and swelling.

Figure 8 shows the optical transmittance of the pristine PC, chemically treated specimen, and plasma spray treated specimen in visible regions between 400 and $700 \mathrm{~nm}$. In the pristine PC of Fig. 8(a), the optical average transmittance in visible regions between 400 and $700 \mathrm{~nm}$ was $88.2 \%$ and the chemical treated specimen was shown to have low optical transmittance by increase of reflective haze due to changes in surface roughness. Figure 8(b) shows the optical transmittance with changes acetone concentrations in the primary chemical treatment and plasma spray treated specimen measured at $550 \mathrm{~nm}$. In the case of primary chemical treatment, it can be seen that as the amount of acetone increases, the degree of swelling on the surface increases, resulting in a decrease dramatically in the optical transmittance due to increased surface roughness. It was also found that the variation in surface roughness due to changes in acetone concentrations in the primary chemical treatment have increased the degree of improvement due to plasma treatment. Therefore, the difference between optical transmittance compared to the primary treatment sample was increase.

\section{Conclusions}

Using the PC polymer sheet, surface treatment using primary chemical surface treatment and secondary plasma spray, the effects on surface roughness and variation in optical characteristics were identified. The primary chemical treatment did not significantly affect changes in the chemical composition of the surface, but the surface roughness was influenced by swelling. Although plasma spray treatment affects surface composition changes, it has a small effect on surface structure changes. Secondary plasma treatment after primary chemical treatment has allowed control of changes in surface structure. At the same time after the first chemical treatment, the secondary plasma treatment was easy to control of optical properties by changing the surface structure as well as the surface properties due to changes in chemical composition by surface cross-linking reaction.

\section{Acknowledgements}

This work was supported by the Shinhan University Research Fund, 2018.

\section{References}

[1] X. Zhu, H. E. Loo, and R. Bai, J. Membr. Sci. 436, 47 (2013).

[2] E. Gogolides, K. Ellinas, and A. Tserepi, Microelectron. Eng. 132, 135 (2015).

[3] T. Liu and C.-J. Kim, Science 346, 1096 (2014).

[4] M. Laurenti, S. Bianco, M. Castellino, N. Garino, A. Virga, C. F. Pirri, and P. Mandracci, ACS Appl. Mater. Interfaces 8, 8032 (2016).

[5] E. Liston, L. Martinu, and M. Wertheimer, J. Adhesion Sci. Technol. 7, 1091 (1993).

[6] S. Dahl, D. Rats, J. von Stebut, L. Martinu, and J. E. KlembergSapiela, Thin Solid Films 355-356, 290 (1999).

[7] U. Schulz, P. Munzert, and N. Kaiser, Surf. Coat. Technol. 142144, 507 (2001).

[8] H. Seo, S. J. Cho, and J. H. Boo, Appl. Sci. Converg. Technol. 23, 44 (2014).

[9] D. R. Paul and L. M. Robeson, Polymer 49, 3187 (2008).

[10] S. Sarva and M. C. Boyce, J. Mechan. Mater. Struct. 2, 1853 (2007).

[11] P. S. Brown and B. Bhushan, Sci. Rep. 6, 21048 (2016).

[12] B. J. Jeon, S. Lee, and J. K. Lee, Surf. Coat. Technol. 202, 1839 (2008).

[13] G. H. Kim and B. J. Jeon, Appl. Sci. Converg. Technol. 25, 133 (2016). 